\title{
The effects of a brain-friendly diet on metabolic and physiological parameters and cognitive performance (Brave study)
}

\author{
Sanna-Maria Hongisto ${ }^{1}$, Marika Laaksonen ${ }^{2}$, Noora Kanerva ${ }^{3}$, Johanna Koponen ${ }^{3}$, \\ Juuso Parkkinen ${ }^{3}$, Emmi Tikkanen ${ }^{3}$, Juhani Sibakov², Jussi Loponen ${ }^{2}$, Heli Diaz ${ }^{1}$, \\ Leila Fogelholm ${ }^{1}$, Jukka Rantala ${ }^{4}$ and Harri Lindholm ${ }^{5}$ \\ ${ }^{1}$ Fazer Food Services, Helsinki, Finland, \\ ${ }^{2}$ Fazer Lab Research Team, Oy Karl Fazer Ab, Helsinki, Finland, \\ ${ }^{3}$ Nightingale Health Oy, Helsinki, Finland, \\ ${ }^{4}$ Nokia Technologies Oy, Espoo, Finland and \\ ${ }^{5}$ Nokia Bell Labs, Espoo, Finland
}

\begin{abstract}
Cognitive performance is related to lipid and glucose metabolism and metabolic activation that are regulated by diet. Further, there is good evidence to support that change in lifestyle, for instance in diet, may promote cognitive health. However, vast majority of the existing studies have focused on children or older adults. We studied the effects of a brain-friendly diet (Brainfood) on metabolic and physiological parameters and cognitive performance in office workers at assumed metabolic risk. We conducted a diet-switch, 4-week intervention study on 84 volunteers with elevated plasma LDL levels in pre-screening. The brainfood diet was planned to be optimal for metabolism and cognitive function; special attention was paid for regular meal frequency, a well-balanced light lunch at work and optimised recipes ensuring quality of carbohydrates and fat, and sufficient protein amount. The control diet was a typical western diet. Breakfast supplies, lunch and afternoon snacks were provided to participants, and they received nutritional counselling. Participants' sociodemographic characteristics, such as age and sex, were queried in the beginning of the intervention. Plasma samples were collected at the end of the 2-week lead-in period, and control and intervention periods. The samples were analysed using high-throughput nuclear magnetic resonance (NMR) spectrometry for quantification of lipoprotein subclasses and particle sizes, cholesterol, glycerides, phospholipids, apolipoproteins, fatty acids, amino acids, ketone bodies, fluid balance, glycolysis and inflammation markers. Participants completed a 3-day food diary at the end of the 2-week lead-in period, and control and intervention periods. Anthropometric measurements (height and weight) were measured at the same timepoints by a study nurse. Physical activity was instructed to be maintained at the pre-study level during the whole study period and it was monitored by using a 3-axial accelerometer (Active style Pro HJA, Omron, Japan). Cognition was measured with standardized neuropsychological tests (Bourdon-Wiersma, FAT, N-Back, NASA-TLX, KSS, RAVLT, Stroop, WMS-III, task-switching) under a psychologist's supervision and as tablet, PC or online tests. Brainfood reduced saturated fat and salt intakes and increased polyunsaturated fat, fibre, vitamin C and D, iron and magnesium intakes. Favourable effects were seen in the atherogenic lipid measurements, such as fatty acids, LDL, IDL and small VLDL, as well as fatty acids, phospholipids and glutamine. Intervention did not affect the physiological or cognitive parameters except for a decrease in inaccuracy of visual attention $(p=0.027)$. In conclusion, the Brainfood diet could be recommended for office workers as a healthy diet following Nordic nutrition recommendations.
\end{abstract}

\section{Conflict of Interest}

SM. Hongisto, M. Laaksonen, Juhani Sibakov, Jussi Loponen, Heli Diaz and Leila Fogelholm are employees of Oy Fazer Ab, Finland. N. Kanerva, J Koponen, J. Parkkinen, and E. Tikkanen are shareholders and employees of Nightingale Health, Ltd - a acompany offering nuclear magnetic resonance-based metabolic profiling. J. Rantala is employee of Nokia Technologies Oy, and Harri Lindholm is employee of Nokia Bell Labs. 